\title{
Polar and alpine microbiology in a changing world
}

\section{Authors/Editors: John C. Priscu, Johanna Laybourn-Parry, Max Häggblom}

This is the peer reviewed version of the following article: Priscu, John C., Johanna Laybourn Parry, and Max Häggblom. "Polar and alpine microbiology in a changing world." FEMS microbiology ecology 89, no. 2 (2014): 209-210. . which has been published in final form at http:// dx.doi.org/10.1111/1574-6941.12371. This article may be used for non-commercial purposes in accordance With Wiley Terms and Conditions for self-archiving.

Priscu, John C., Johanna Laybourn - Parry, and Max Häggblom. "Polar and alpine microbiology in a changing world." FEMS microbiology ecology 89, no. 2 (2014): 209-210.

http://dx.doi.org/10.1111/1574-6941.12371.

Made available through Montana State University's ScholarWorks

scholarworks.montana.edu 


\section{Polar and alpine microbiology in a changing}

\section{world}

\author{
John C. Priscu (Issue Editor): \\ Department of Land Resources and Environmental Science \\ Montana State University \\ Bozeman, MT, USA \\ e-mail: jpriscu@montana.edu
}

\author{
Johanna Laybourn-Parry (Issue Editor): \\ School of Geographical Sciences \\ University of Bristol \\ Bristol, UK, \\ e-mail: jo.laybourn-parry@bristol.ac.uk
}

\author{
Max Haggblom (Chief Editor): \\ Department of Biochemistry and Microbiology \\ Rutgers University \\ New Brunswick, N], USA \\ e-mail: haggblom @ aesop.rutgers.edu
}

High altitude and high latitude regions on Earth are experiencing rapid changes in climate. Ecological impacts resulting from these changes are now being observed at all ecosystem levels and larger deviations and more significant impacts are anticipated in the future. Satellite data show dramatic reductions in the extent and thickness of sea ice at both poles, and rising temperatures are causing alpine glaciers worldwide to shrink in area and volume. By virtue of their relatively rapid growth rates and metabolic diversity, we can expect microorganisms to be the first responders to fluctuating climatic conditions. Because microorganisms are keystone players in elemental transformations, variations in their abundance and diversity will initiate a cascade of impacts throughout entire ecosystems. Clearly, knowledge of the distribution, biodiversity and functional roles of microorganisms inhabiting polar and alpine environments is essential to our understanding of ecosystem processes in a changing climate.

The continued warming and diminishing extent of the polar and alpine cryosphere will be a major driver of the microbially mediated processes occurring in these environments. We can expect to see changes in biodiversity, ecosystem and organismal stoichiometry, amplification of certain trophic levels, and a change in genomic connectivity among ecosystem components as the result of a warming cryosphere. Eventually, the climate-driven changes occurring in polar and alpine systems will reach critical thresholds or tipping points, if they have not already, that will bring them into a new state or level of activity. Consequently, it is imperative that we identify key connections and feedbacks resulting from the interactions between the global climate system and the microbial ecosystems that inhabit Earth's cryosphere. Particularly important is our understanding of the genetic, taxonomic, and functional aspects of microbial biodiversity in a changing world. There is a need for a holistic, interdisciplinary systems approach to the microbiology of polar and alpine regions if we are to truly identify microbial responses to climate change.

Understanding the adaptability of microorganisms on Earth to changing climate will also provide us with important insights in our search for microbial life beyond Earth. Given that life has been microscopic throughout most of Earth's history and even today microorganisms dominate our planet in the most diverse and extreme environments, it is reasonable to presume that if life exists beyond Earth, it most likely would have started as prokaryotic microorganisms and may still remain in this stage of evolution today. As our active exploration of space begins its sixth decade, we have, for the first time in the history of humanity, the tools and techniques to probe the profound question of microbial habitability on worlds beyond Earth. The icy moons of the outer Solar System, worlds like Europa and Enceladus that orbit Jupiter and Saturn, respectively, are covered by ice and harbor subsurface liquid water oceans that contain many times the volume of liquid water found on Earth. These oceans are there today and have probably persisted for much of the history of the solar system making them ideal environments in which to search for microbial life beyond Earth and which to examine a possible second, independent origin of life. If microbial life were discovered on the icy worlds of our outer solar system, we could then begin a rigorous cross-comparison to investigate the conditions leading to the emergence of life on both worlds and to the role that the cryosphere has in the evolution of these microorganisms.

These are indeed exciting times to be a microbial ecologist. An understanding of contemporary dynamics of microorganisms on our planet, and possibly elsewhere, will provide us with the background necessary to understand the complex interactions that will occur as climate changes in our polar and alpine regions. New and emerging technologies in the field of genomics will allow us to study the structure, content, and evolution of genomes including the analysis of the expression and function of both gene and proteins. Next generation sequencing in concert with new developments in bioinformatics will allow genomic data to be converted into a format that can be included in ecosystem models that address evolution, diversity, biogeography, biogeochemistry, and metabolic capacity in response to environmental change. When coupled with remote sensing, in situ instrumentation, and long-term observatories, genomic data from specific cryospheric environments can be used to evaluate ongoing and potential impacts of polar and alpine climate forcing on all of the ecosystems on Earth, including the interconnectivity they have with humans. The global impact of rapidly changing climate make polar and alpine environments a fundamental concern for science and society. 
Advances in our understanding of the microbiology of polar and alpine environments have been presented and discussed through a series of conferences focusing on polar and alpine microbiology. The first Polar and Alpine Microbiology Conference was held in Rovaniemi, Finland in 2004. Subsequent conferences were in Innsbruck, Austria (2006), Banff, Canada (2008), Ljubljana, Slovenia (2011) and the latest one Big Sky, Montana (2013). These conferences have provided a multidisciplinary forum to explore emerging areas in the field, and provide opportunities for the exchange of ideas and building of collaborations. The undeniable realization that polar and alpine environments are being influenced significantly and perhaps irreversibly by rapid climate change led us to the theme for the Big Sky conference: 'Discovery in a Changing World'. The Big Sky conference brought together more than 140 international researchers and included 54 oral presentations and 70 posters. Following an opening lecture that focused on more than 20 years of ecosystem response in Antarctic marine waters, the conference was divided into seven sessions. The first three sessions focused on linking the polar and alpine cryosphere to other ecosystems, identifying first responders to climate change, and addressing potential thresholds and tipping points in the microbial world related to change. Sessions four and five addressed the emerging field of subglacial microbiology and examined the evolution of psychrophilic microorganisms. Session six focused on biodiversity, ecosystem function and environmental change in the past, present, and future, and Session seven took us beyond earth to discuss potential microbial life on other icy worlds using Earth's subzero biosphere as a model system.

This thematic issue of FEMS Microbiology Ecology includes 22 peer-reviewed manuscripts based on presenta- tions given at the 2013 Big Sky Conference as well as other submissions received. The manuscripts present new information on the distribution, biodiversity and functional role of microorganisms in marine, freshwater, terrestrial, and glacial environments from high latitude and high altitude environments, all of which are being impacted significantly by climate warming. Collectively, the information contained in these manuscripts reveals that not all of the details fit in perfectly; if they did we would have to question the biocomplexity of the microbial world. The information does provide a new baseline from which to launch future research efforts and make new discoveries in our changing world. 